sunumasmall

DOI: $10.1002 / \mathrm{smll} .200800353$

\title{
Real-time Tracking of Superparamagnetic Nanoparticle Self-assembling
}

P. Siffalovic, E. Majkova, L. Chitu, M. Jergel, S. Luby, I. Capek, A. Satka, A. Timmann, and S. V. Roth*

[*] Dr. P. Siffalovic, Dr. E. Majkova, MSc. L. Chitu, Dr. M. Jergel, Prof. S. Luby

Institute of Physics, Slovak Academy of Sciences

Dubravska cesta 9, 84511 Bratislava (Slovakia)

E-mail: peter.siffalovic@savba.sk

Prof. I. Capek

Polymer Institute, Slovak Academy of Sciences

Dubravska cesta 9, 84511 Bratislava (Slovakia)

Dr. A. Satka

International Laser Center

Dubravska cesta 9, 84511 Bratislava (Slovakia)

Dr. A. Timmann, Dr. S. V. Roth

HASYLAB/DESY

Notkestrasse 86, 22603 Hamburg (Germany)

Keywords: self-assembly, nanoparticles, X-ray scattering

We report on a real-time observation of the spontaneous self-assembly process of superparamagnetic nanoparticles in a fast drying colloidal drop. The grazing-incidence small angle X-ray scattering (GISAXS) technique was employed for an in-situ tracking of the reciprocal space with a 3 ms delay time between subsequent frames delivered by a new generation of X-ray cameras. A focused synchrotron beam and sophisticated sample oscillations opened the possibility to relate the dynamic reciprocal and direct space features and to localize the self-assembling. In particular, no measurable nanoparticle ordering inside the evaporating drop and near-surface region down to $90 \mu \mathrm{m}$ drop thickness was found. Scanning through the shrinking drop contact line indicates the start of self-assembling near the drop three-phase interface in accord with theoretical predictions. The results obtained have 


\section{wave \\ saments small}

direct implications for establishing the self-assembly process as a routine technological step in preparation of new nanostructures.

\section{Introduction}

Latest advances in nanochemistry ${ }^{[1]}$ made possible the synthesis of highly monodisperse colloidal nanoparticles. In particular, magnetic nanoparticles ${ }^{[2]}$ become increasingly important in applied materials science ranging from bio-applications in targeted drug delivery ${ }^{[3]}$ to utilization in emerging spintronic devices ${ }^{[4]}$. Magnetic nanoparticles offer a possibility of quantized electron tunneling and additional spin blockade. An ordered array of such nanoparticles can be used as a natural double-tunnel barrier in novel tunnel magnetoresistance devices ${ }^{[5]}$ or spin torque nanooscillators ${ }^{[6]}$. These well correlated nanoparticle templates result from the process of spontaneous nanoparticle self-assembly ${ }^{[7]}$. The bottom-up approach ${ }^{[8]}$ presents a fast and cost-effective way of fabrication of nanoparticle monolayers.

The dynamics of noble metal nanoparticle self-assembling in an evaporating colloidal drop has been investigated by numerous groups ${ }^{[9-12]}$. As the origin of the self-assembling, the contact line region of the dewetting colloidal drop was identified. On the other hand, the tendency of gold nanoparticles to self-assembling at the drop surface has been stimulated and experimentally verified by adding an excess of surfactant molecules ${ }^{[11]}$. Mostly vacuum-free direct space techniques like the visible light microscopy were utilized to observe the formation of self-assembled nanoparticle templates on surfaces. However, this technique cannot address the nanoparticle position correlations within the ordered nanoparticle domains during the drop evaporation. Here, the grazing-incidence small angle X-ray scattering (GISAXS) was recognized to be a promising tool for the real-time observation of 


\section{small}

self-assembling in the reciprocal space ${ }^{[13]}$. Whereas a low evaporation rate facilitated such GISAXS observations for the water-based noble metal colloidal solutions ${ }^{[14,15]}$, the use of non-polar rapidly evaporating solvents for superparamagnetic nanoparticle colloids calls for monitoring techniques with several milliseconds time response only ${ }^{[16]}$. We developed scanning GISAXS schemes where the probing focused X-ray beam scans a horizontally or vertically oscillating sample across the regions of interest - total drop height or drop contact line. The spatial smearing of the scattering volume is minimized by a suitably selected scanning velocity and fast data acquisition time.

\section{Results and Discussion}

The iron oxide nanoparticles were synthesized by a high-temperature solution phase reaction of metal acetylacetonates $\left(\mathrm{Fe}(\mathrm{acac})_{3}\right)$ with 1,2-hexadecanediol, oleic acid, and oleylamine in phenyl ether as described elsewhere ${ }^{[17]}$. The nanoparticles were dispersed in toluene. The average nanoparticle diameter of $6.4 \pm 0.6 \mathrm{~nm}$ was determined by scanning electron microscopy ${ }^{[16]}$. The half-metallic nanoparticle is a single crystal with the dimensions matching the size of the nanoparticle core as revealed by X-ray diffraction ${ }^{[16]}$. The nanoparticle organic shell - surfactant surrounding the crystalline core of nanoparticles has a stabilizing function. The nanoparticles are superparamagnetic at room temperature with the blocking temperature of $22 \mathrm{~K}$.

The GISAXS experiments were conducted at BW4 beamline ${ }^{[18]}$, HASYLAB. A $5 \mu 1$ drop of colloidal nanoparticles was applied on a clean silicon substrate at room temperature. The Figure 1 shows the scanning electron microscopy image of nanoparticle array after solvent evaporation. Evaporation process of the rapidly evaporating solvent has three distinctive stages. In the first stage, the drop wets the substrate and the drop contact line reaches its maximum diameter. The second stage is dominated by a linear decrease of the 


\section{small}

drop mass accompanied by a shrinking of the drop contact line. The polished silicon substrate prevents from the pinning of the contact line. However, the nanoparticles themselves serve as pinning centers which can be observed as a gradual stick-slip motion of the drop contact line. The third stage starts when the evaporation-driven surface tension instability initiates random drop movement along the substrate. In this study, we addressed mainly the second evaporation stage that is responsible for the formation of self-assembled nanoparticle arrays. The beginning of the third evaporation stage is also observed and completes our discussion enabling us an exhaustive and coherent picture of the nanoparticle ordering.

The geometry of the GISAXS vertical scanning scheme is shown in Figure 2. The incident X-ray beam with the wave vector $\vec{k}_{i}$ is scattered. The $q_{y}, q_{z}$ components of the momentum transfer vector $\vec{q}$ are aligned as shown in Figure 2 while the $q_{x}$ component, which is normal to them, is not shown. The scattered radiation is measured and analyzed as a two-dimensional function in the $q_{y}-q_{z}$ plane. The substrate with the applied evaporating drop oscillates vertically across the incident beam with a constant velocity. Three major scattering zones are distinguished when considering e.g. upward movement of the sample. In the zone $\mathrm{Z} 0$, the sampling beam is located above the drop surface and only a background scattering is recorded. The limits of the zone $\mathrm{Z} 1$ are defined by the first contacts of the beam with the drop surface and the substrate. Here, the scattering is due solely to the drop near-surface region and drop volume. The zone Z2 produces a combined scattering by the drying drop and the substrate. The zone Z1 plays the major role in this study since it gives an unambiguous determination of the scattering volume.

Figure 3a shows the final GISAXS pattern recorded after a complete solvent evaporation. The self-assembled nanoparticle areas are manifested by a side maximum at $q_{y} \sim-0.82 \mathrm{~nm}^{-1}$. The central part of the scattering pattern is not obstructed by the conventional specular beamstop as the high saturation counting rate of $10^{6}$ photons/s permits the 
observation of the specularly reflected beam. The time-resolved monitoring of this reflectivity also provides useful information on the scattering volume (see further). The streak of the scattered radiation along $q_{z}$ at $q_{y}=0$ in Figure $3 \mathrm{a}$ is the radiation scattered by the substrate roughness and dried nanoparticle layer which is usually measured in the $q_{x}$ - $q_{z}$ plane (coplanar geometry) as a detector scan. The visibility of the projection of the detector scan onto $q_{y}-q_{z}$ plane in Figure 3a is due to the non-zero scattering component $q_{x}$ which is, however, more than one order of magnitude smaller than $q_{y}$. Therefore, $q_{x}$ will be omitted in the analysis of the non-coplanar GISAXS. In the Born approximation ${ }^{[19]}$, the scattered radiation is described by multiplication of the squared modulus of the nanoparticle form factor and the interference function of the nanoparticle distribution. The nanoparticle form factor and interference function are given by Fourier transform of the averaged electron density of the nanoparticle and the autocorrelation function of the nanoparticle distribution, respectively. For planar samples measured in reflection geometry like GISAXS and exhibiting the total external reflection, the distorted-wave Born approximation ${ }^{[20,21]}$ (DWBA) must be applied when observing the scattered radiation close to the critical angle. The DWBA corrects the simple Born approximation for multiple scattering events near the critical angle. The GISAXS pattern simulated within the DWBA and paracrystal model ${ }^{[22]}$ is shown in Figure $3 \mathrm{~b}$. Only diffusely scattered radiation was calculated, hence, the specular beam reflected by the substrate cannot be seen in the simulation. The following simulation parameters were used: nanoparticle radius $=3.4 \pm 0.3 \mathrm{~nm}$, interparticle distance $=7.5 \pm 1 \mathrm{~nm}$, lateral correlation length $=87 \mathrm{~nm}$. The measured GISAXS pattern supported by the simulation proves that the substrate is covered with an ordered nanoparticle monolayer after solvent evaporation as verified also by scanning electron microscopy ${ }^{[16]}$.

The time-resolved tracking of the self-assembly before the complete solvent evaporation is the core of our study. In order to visualize the self-assembling, the data were 
processed into two different kinds of maps. In a so called $t$ - $q_{y}$ map (Figure 4a), the region between the two dotted horizontal lines at $q_{z}=0.22$ and $0.4 \mathrm{~nm}^{-1}$ depicted in Figure 3a was integrated and plotted as a function of time. In a so called $t-q_{z}$ map (Figure $4 \mathrm{~b}$ ), integration for $q_{y}$ between $\pm 0.02 \mathrm{~nm}^{-1}$ (the region between the two vertical dashed lines in Figure 3a) was performed and plotted as a function of time. Briefly, the $t-q_{y}$ map describes the temporal evolution of the $q_{y}$ component of the scattering vector near the critical exit angle and the $t-q_{z}$ map describes the temporal evolution of what is called above the detector scan. The instances when the sampling $\mathrm{X}$-ray beam comes into contact with the drop surface ( $\mathrm{Z} 0 \rightarrow \mathrm{Z} 1$ transition) or when it leaves the drop ( $\mathrm{Z} 1 \rightarrow \mathrm{Z} 0$ transition) are clearly visible as an enhanced scattering along $q_{y}$ direction in the $t-q_{y}$ map for the early evaporation stage (time less than approx. $60 \mathrm{~s}$ ). Between these extremes, more absorbed and weaker radiation coming from the zone $\mathrm{Z} 2$, where the X-ray beam starts to interact with the substrate, can be seen. The $\mathrm{Z} 0 \rightarrow \mathrm{Z} 1$ and $\mathrm{Z} 1 \rightarrow \mathrm{Z} 0$ transitions are marked also by significant scattering in the $t-q_{z}$ map while the detector scans in between produced by the substrate scattering in the zone $\mathrm{Z} 2$ are less prominent. This behavior is reversed at later evaporation stages (after approx. $60 \mathrm{~s}$ ) where the main scattering stems from the zone $\mathrm{Z} 2$ and the substrate scattering plays the major role. It can be observed in the $t$ - $q_{y}$ map as a strong scattering signal surrounded by a lower one coming from the zone Z1. Similarly in the $t-q_{z}$ map, the detector scans resulting from the $\mathrm{Z} 0 \rightarrow \mathrm{Z} 1$ and $\mathrm{Z} 1 \rightarrow \mathrm{Z} 0$ transitions are decreasing in intensity in favour of the detector scans originating from the substrate scattering in the zone Z2. The temporal approaching of the occurrence of the detector scans (i.e. $\mathrm{Z} 0 \rightarrow \mathrm{Z} 1$ and $\mathrm{Z} 1 \rightarrow \mathrm{Z} 0$ transitions) belonging to one sample oscillation cycle indicates the thinning of the evaporating colloidal drop.

The degree of the nanoparticle ordering in the self-assembled monolayer affects the number, width and intensity of the GISAXS maxima. We characterize the intensity of the first GISAXS maximum by a so-called partially integrated scattering (PIS) obtained by an 


\section{wave \\ sameress small}

integration within the square region limited by the dotted lines in Figure 3a. The temporal evolution of PIS was extracted from the $t-q_{y}$ map (Figure 4a) by an integration between the two horizontal dotted lines which correspond to the values $q_{y}=0.65$ and $0.95 \mathrm{~nm}^{-1}$. The time-resolved reflectivity was obtained by integrating the GISAXS frames within a small $q_{y}-q_{z}$ area where the specularly reflected beam hit the X-ray camera. The temporal plots of PIS and specular reflectivity are shown in Figure 5. The PIS signal originating from the zone Z1 is almost constant which indicates that number of scattering centers (nanoparticles) and their interference function do not change with time. The PIS signal corresponding to the zone $\mathrm{Z} 2$ becomes clearly visible for evaporation times longer than approx. $60 \mathrm{~s}$. This signal comes from the already dried areas on the substrate covered with the self-assembled nanoparticle monolayer and steadily increases before leveling-off in the very last stage. The time-resolved reflectivity curve consists of a series of dominant peaks, each surrounded by two satellites. The satellite peaks occur at the $\mathrm{Z} 0 \rightarrow \mathrm{Z} 1$ and $\mathrm{Z} 1 \rightarrow \mathrm{Z} 0$ transitions. Their intensities do not change because of the constant reflectivity of the air/drop interface. The central peaks come from the total reflection at the drop/substrate interface $(\mathrm{Z} 1 \rightarrow \mathrm{Z} 2$ transition). The gradual increase of their intensity is associated with the shortening absorption path in the evaporating drop. The critical angle for total reflection of the toluene solvent and silicon substrate are approx. $0.12^{\circ}$ and $0.2^{\circ}$, respectively. Therefore the satellite peaks are weaker than the central ones, not being produced by the total reflection at $0.2^{\circ}$ angle of incidence.

The observed scattered intensity as a function of the scattering vector component $q_{y}$ is given by multiplication of the nanoparticle form factor and interference function in the first approximation. We integrated intensity in the $t-q_{y}$ map (Figure 4a) along the temporal axis within the zone $\mathrm{Z} 1$ for every particular oscillation cycle to obtain the scattering curves. Three of them corresponding to the initial, intermediate and final evaporation stages are shown in 


\section{somins small}

Figure 6a. All these scattering curves coming from the drop near-surface and interior regions show the absence of any constructive interference. We used the formula

$$
I\left(q_{y}\right)=A+B\left|F\left(q_{y}\right)\right|^{2} S\left(q_{y}\right)
$$

to fit the measured curves. The $F\left(q_{y}\right)=4 \pi r^{3}\left[\sin \left(q_{y} r\right)-q_{y} r \cos \left(q_{y} r\right)\right] /\left(q_{y} r\right)^{3}$ is the form factor of a full sphere with the radius $r, S\left(q_{y}\right)$ is the interference function, $A$ and $B$ are offset and scaling constants of the experimental data, respectively. A constant interference function $S\left(q_{y}\right)$ equal to unity was applicable in all fits and provided a good match between the measured data and fit. Such an interference function implies no correlations between the nanoparticle positions which is characteristic for a diluted system of non-interacting nanoparticles $^{[19]}$. To quantify the goodness of fit, we used the adjusted coefficient of determination $^{[23]}$ (also known as adjusted $\mathrm{R}^{2}$ ) which is a suitable measure of goodness of different multiparameter data fits and allows for their direct comparison. The adjusted coefficient of determination of $I\left(q_{y}\right)$ fits for the zone Z1 throughout the whole evaporation stage is plotted in Figure $6 \mathrm{~b}$. It increases slightly with time and confirms the assumption of no lateral correlations between the nanoparticles near the surface and inside the drop. The temporal evolution of the scaling parameter $B$ (Figure 6c) is nearly constant with an exception at the end where a single oscillation appears. The nearly constant value of $B$ indicates a nearly constant number of the scattering nanoparticles near the surface and inside the drop while the final oscillation is connected with a sudden escape of the evaporating drop from the probed volume. These rapid drop movements in the final evaporation stage are initiated by the evaporation-driven surface tension instability on polished substrates without intrinsic pinning centers. The drop thickness evolution was extracted from the $\mathrm{Z} 0 \rightarrow \mathrm{Z} 1$ and $\mathrm{Z} 1 \rightarrow \mathrm{Z} 0$ transitions within one sample oscillation cycle as observed in the $t$ - $q_{y}$ map (Figure $4 \mathrm{a}$ ). The elevation of the drop surface above the substrate is shown in Figure 6d. The drop 
thickness decreases from approx. $150 \mu \mathrm{m}$ to $90 \mu \mathrm{m}$ which is further limited by the vertical size of the sampling X-ray beam. The abrupt oscillation in the final evaporation stage is another confirmation of the drop movement and correlates well with the oscillation of the $B$ parameter in Figure 6c. The temporal coincidence of the $B$ parameter and drop thickness oscillations is evident. The PIS obtained from the $t$ - $q_{y}$ map (Figure 4a) by an integration inside the zone Z2 only is shown in Figure 6e. The increasing PIS signal is assigned to the enlarging of the already dried surface covered with the self-assembled monolayer. The analysis performed implies that the self-assembly starts in the vicinity of the drop contact line rather than near the surface or inside the drop where no self-assembled clusters were found.

To support the assumption about the self-assembling near the contact line, we employed also a horizontal scanning scheme as shown in Figure 7. Here, the sample oscillates horizontally across the incoming X-ray beam which intersects periodically the shrinking drop contact line during the solvent evaporation. The scanning velocity was adapted to the exposure and read-out times of the X-ray camera in order to minimize the effect of the spatial smearing on the scattering volume sampled by the X-ray beam. The $t$ - $q_{y}$ map measured in the horizontal scanning mode is shown in Figure 8a. At the beginning, periodic stripes of an enhanced intensity along the $q_{y}$ axis with the maximum at $q_{y} \sim 0.82 \mathrm{~nm}^{-1}$, coming from the already dried regions of the self-assembled monolayer of nanoparticles and adjacent low-absorbing volume of the drop close to the three-phase contact line, are observed. In between, the sampling X-ray beam enters the drop and is strongly suppressed in intensity by the drop absorption, showing no correlations between the nanoparticle positions. The peak at $q_{y} \sim 0.82 \mathrm{~nm}^{-1}$ persists up to the complete drop evaporation which indicates a well-ordered and stable nanoparticle monolayer. The temporal PIS plot extracted from the $t$ - $q_{y}$ map is compared with the time-resolved specular reflectivity curve in Figure 8b. The minima in the PIS plot and reflectivity are correlated in the early evaporation stages as both are observed 


\section{small}

when the probing X-ray beam is strongly attenuated inside the drop. As the beam crosses the three-phase contact line and leaves the drop, an enhanced scattering in the PIS plot is observed, followed by a significant increase of the reflectivity signal. The opposite takes place when the beam enters the drop. The reflectivity maxima correspond to extreme positions of the horizontal scan when the maximum area of the bare substrate adjacent to the drop is hit by the probing beam. In between, the reflectivity comes mainly from the already dried areas of the colloidal solution with the nanoparticle-related roughness and is thus inherently lower. On the other hand, these areas contribute to the GISAXS signal which is at maximum in the central part of the scan (i.e. in the drop center) when the drop is evaporated completely. Therefore in the final evaporation stages, the PIS signal reaches a steady-state value with a small oscillating part which is in anti-phase with the reflectivity oscillations. Here, the oscillations in the PIS plot and reflectivity are due to a periodically changing ratio between the illuminated areas favoring reflectivity (bare substrate) and GISAXS (dried nanoparticle monolayer). These measurements indicate that behind the shrinking contact line, the self-assembled monolayer is originating. Because no measurable lateral correlations of the nanoparticles near the surface or inside the drop were observed by the vertical scanning, the vicinity of the three-phase drop boundary is the location of the nanoparticle accumulation and self-assembling.

\section{Conclusions}

Summarizing, we employed an original scanning version of the GISAXS technique for a real-time tracking of the fast self-assembling of superparamagnetic nanoparticles dispersed in toluene. No measurable self-assembled clusters were found near the surface or inside the drop. The vicinity of the three-phase drop contact line was identified as the location where the deposition of the self-assembled monolayer starts. The present work is directly relevant for 


\section{Submitted to}

the understanding and practical utilization of self-assembly phenomena in superparamagnetic nanoparticle colloids.

\section{Experimental Section}

The scanning GISAXS measurements were done at BW4 beamline at HASYLAB synchrotron, Hamburg (Germany). The focused X-ray beam of the size $65 \times 35 \mu \mathrm{m}^{2}(\mathrm{HxV})$ and the wavelength of $0.138 \mathrm{~nm}$ hit a silicon substrate at $0.2^{\circ}$ grazing angle of incidence. The X-ray camera PILATUS $100 \mathrm{~K}$ at a distance of $225 \mathrm{~cm}$ from the sample was used. The camera exposure time was set to $25 \mathrm{~ms}$ and the read-out time was $3 \mathrm{~ms}$. The pixel size of the X-ray camera is $172 \times 172 \mu \mathrm{m}^{2}$. The colloidal drop was applied manually on the silicon substrate. The zero of the time scale is related to the activation of the hutch interlocks and has some $30 \mathrm{~s}$ delay with respect to the absolute time scale related to the drop application. In these measurements, the absolute time scale is not relevant. The substrate velocity was set to $40 \mu \mathrm{m} / \mathrm{s}$ with a $200 \mu \mathrm{m}$ total substrate displacement in the vertical scanning mode. The spatial smearing of the scattering volume of approx. $1 \mu \mathrm{m}$ in the vertical scanning mode is negligible when compared to the beam size. The vertical displacement of the scattering pattern on the X-ray camera is of the order of one pixel size and can be neglected as well. In the horizontal scanning mode, the velocity was set to $480 \mu \mathrm{m} / \mathrm{s}$ and the total substrate displacement was $2.5 \mathrm{~mm}$. The spatial smearing of the scattering volume is approx. $14 \mu \mathrm{m}$ which is still smaller than the horizontal beam size.

\section{Acknowledgements}

The work was supported by Scientific Grant Agency VEGA Bratislava, grant no. 2/6030/26, and 2/0047/08, by Centre of Excellence SAS Physics of Information contract no. I/2/2008 and 


\section{sonimustosmall}

by Slovak Research and Development Agency grant Nr. APVV-LPP-0080-06 and APVV0173-06. The support of EU HASYLAB grant RII3-CT-2004-506008, MNT-ERA-2007-009SK and NATO CLG 982748 grant are also acknowledged.

[1] J. Park, K. An, Y. Hwang, J. G. Park, H. J. Noh, J. Y. Kim, J. H. Park, H. M. Hwang, T. Hyeon, Nature Materials 2004, 3, 891-895.

[2] D. L. Huber, Small 2005, 5, 482-501.

[3] L. Zhang, F. X. Gu, J. M. Chan, A. Z. Wang, R. S. Langer, O. C. Farokhzad, Clinical Pharmacology \& Therapeutics 2007, 83, 761-769.

[4] K. Yakushiji, F. Ernult, H. Imamura, K. Yamane, S. Mitani, K. Takanashi, S. Takahashi, S. Maekawa, H. Fujimori, Nature Materials 2004, 4, 57-61.

[5] S. A. Wolf, D. D. Awschalom, R. A. Buhrman, J. M. Daughton, S. von Molnar, M. L. Roukes, A. Chtchelkanova, D. M. Treger, Science 2001, 294, 1488-1495.

[6] S. I. Kiselev, J. C. Sankey, I. N. Krivorotov, N. C. Emley, R. J. Schoelkopf, R. A. Buhrman, D. C. Ralph, Nature 2003, 425, 380-383.

[7] C. B. Murray, C. R. Kagan, M. G. Bawendi, Science 1995, 270, 1335-1338.

[8] S. M. Yang, S. G. Jang, D. G. Choi, S. Kim, H. K. Yu, Small 2006, 4, 458-475.

[9] R. D. Deegan, O. Bakajin, T. F. Dupont, G. Huber, S. R. Nagel, T. A. Witten, Nature 1997, 389, 827-829.

[10]E. Rabani, D. R. Reichman, P. L. Geissler, L. E. Brus, Nature 2003, 426, 271-274.

[11] T. P. Bigioni, X. M. Lin, T. T. Nguyen, E. I. Corwin, T. A. Witten, H. M. Jaeger, Nature Materials 2006, 5, 265-270.

[12]E. Adachi, A. S. Dimitrov, K. Nagayama, Langmuir 1995, 11, 1057-1060. 


\section{sanums small}

[13] G. Renaud, R. Lazzari, C. Revenant, A. Barbier, M. Noblet, O. Ulrich, F. Leroy, J. Jupille, Y. Borensztein, C. R. Henry, J. P. Deville, F. Scheurer, J. Mane-Mane, O. Fruchart, Science 2003, 300, 1416-1419.

[14] S. Narayanan, J. Wang, X. M. Lin, Phys. Rev. Lett. 2004, 93, 135503.

[15] S. V. Roth, T. Autenrieth, G. Grübel, C. Riekel, M. Burghammer, R. Hengstler, L. Schulz, P. Müller-Buschbaum, Appl. Phys. Lett. 2007, 91, 091915.

[16]P. Siffalovic, E. Majkova, L. Chitu, M. Jergel, S. Luby, A. Satka, S. V. Roth, Phys. Rev. B 2007, 76, 195432.

[17] S. Sun, H. Zeng, D. B. Robinson, S. Raoux, P. M. Rice, S. X. Wang, G. Li, J. Am. Chem. Soc. 2004, 126, 273-279.

[18] S. V. Roth, R. Döhrmann, M. Dommach, M. Kuhlmann, I. Kröger, R. Gehrke, H. Walter, C. Schroer, B. Lengeler, P. Müller-Buschbaum, Rev. Sci. Instrum. 2006, 77, 085106.

[19] A. Guinier, A. X-Ray Diffraction, W. H. Freeman and Company, San Francisco and London, 1963.

[20] M. Rauscher, T. Salditt, H. Spohn, Phys. Rev. B 1995, 52, 16855-16863.

[21]P. Muller-Buschbaum, Anal. Bioanal. Chem. 2003, 376, 3-10.

[22] R. Lazzari, J. Appl. Crystallogr. 2002, 35, 406-421.

[23] T. P. Ryan, Modern Engineering Statistics, John Wiley \& Sons, New Jersey, 2007.

Received: ((will be filled in by the editorial staff))

Revised: ((will be filled in by the editorial staff)) Published online on ((will be filled in by the editorial staff)) 


\section{waw \\ suminasus small}

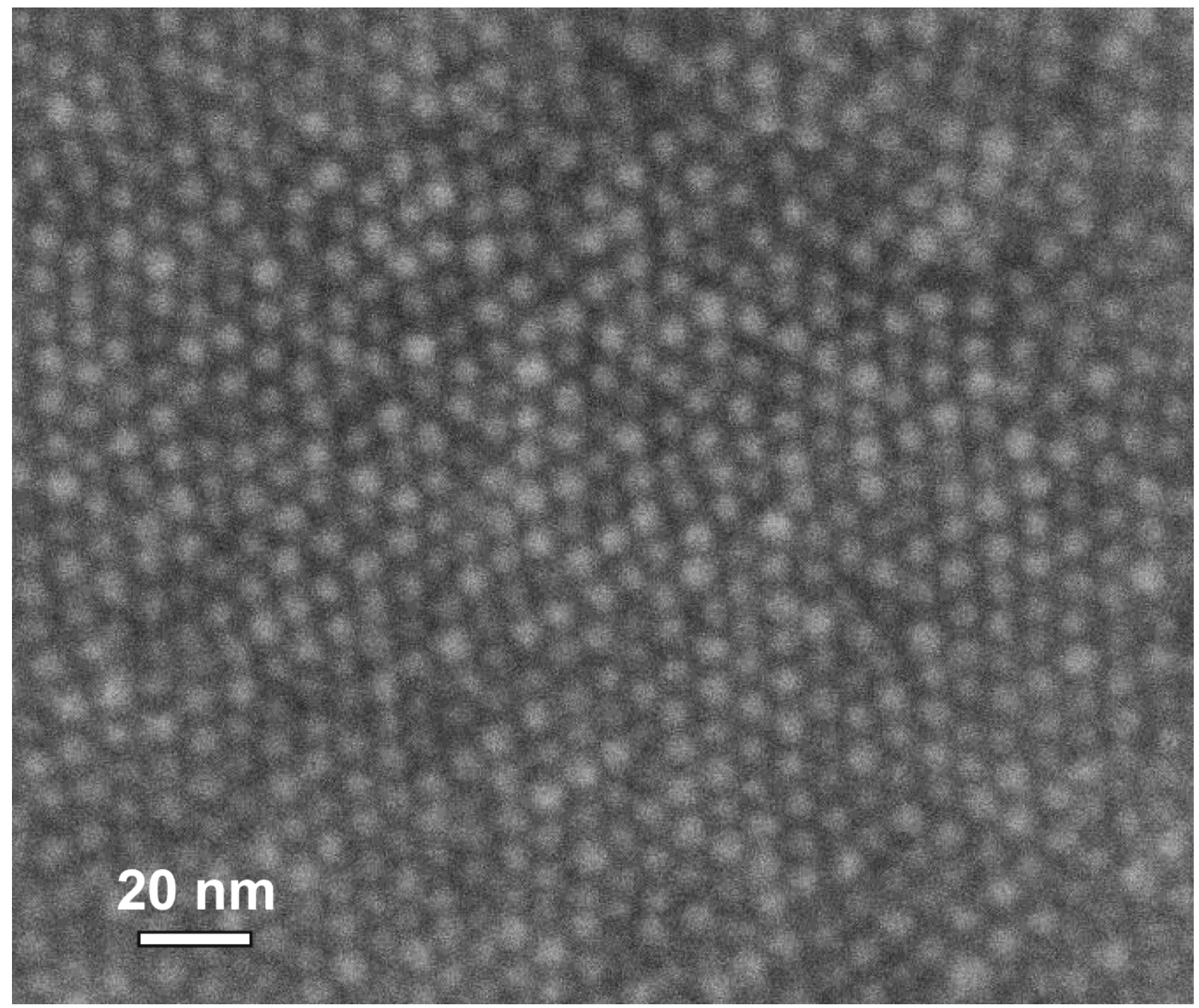

Figure 1. Scanning electron microscope image of an ordered nanoparticle array after solvent evaporation. 


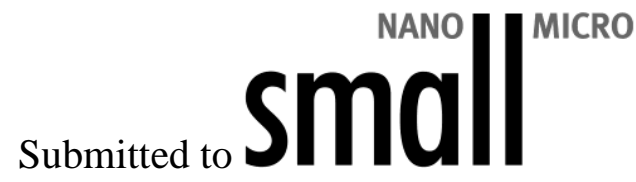

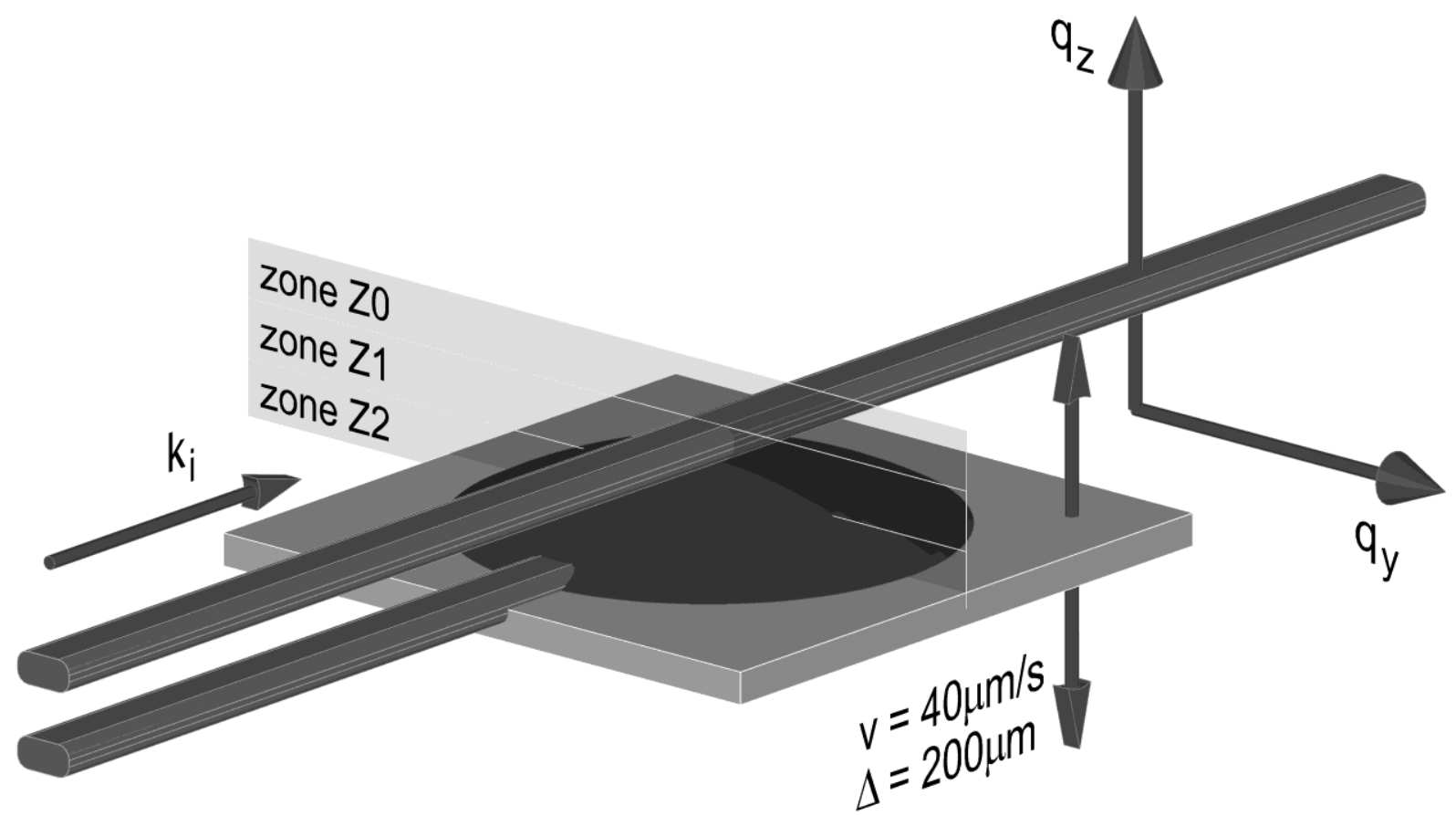

Figure 2. Vertical scanning scheme 


\section{small}

a)

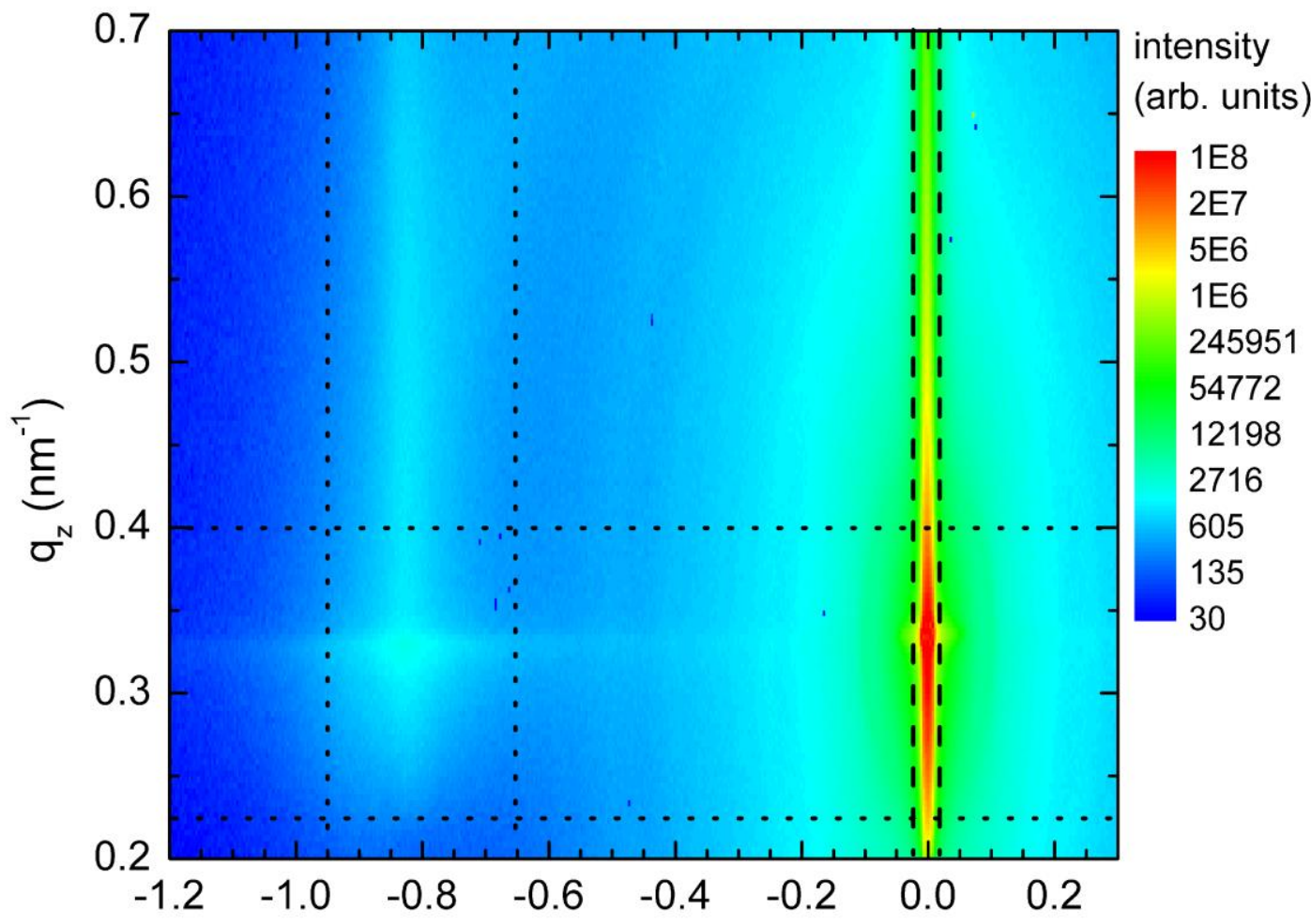

b)

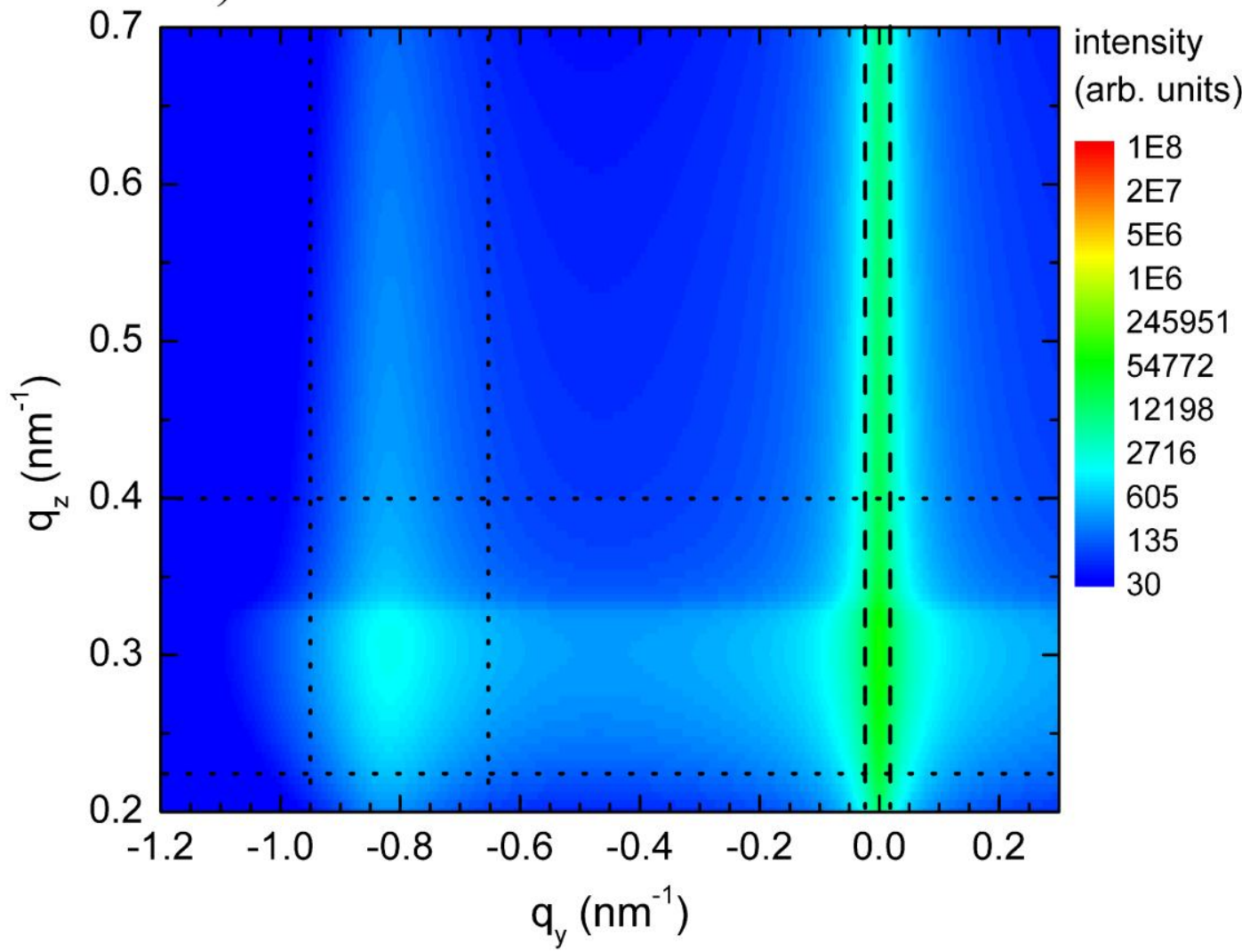

Figure 3. Measured (a) and simulated (b) GISAXS patterns of the self-assembled nanoparticle array 


\section{sominicats Small ${ }^{\text {numo }}$}

a)

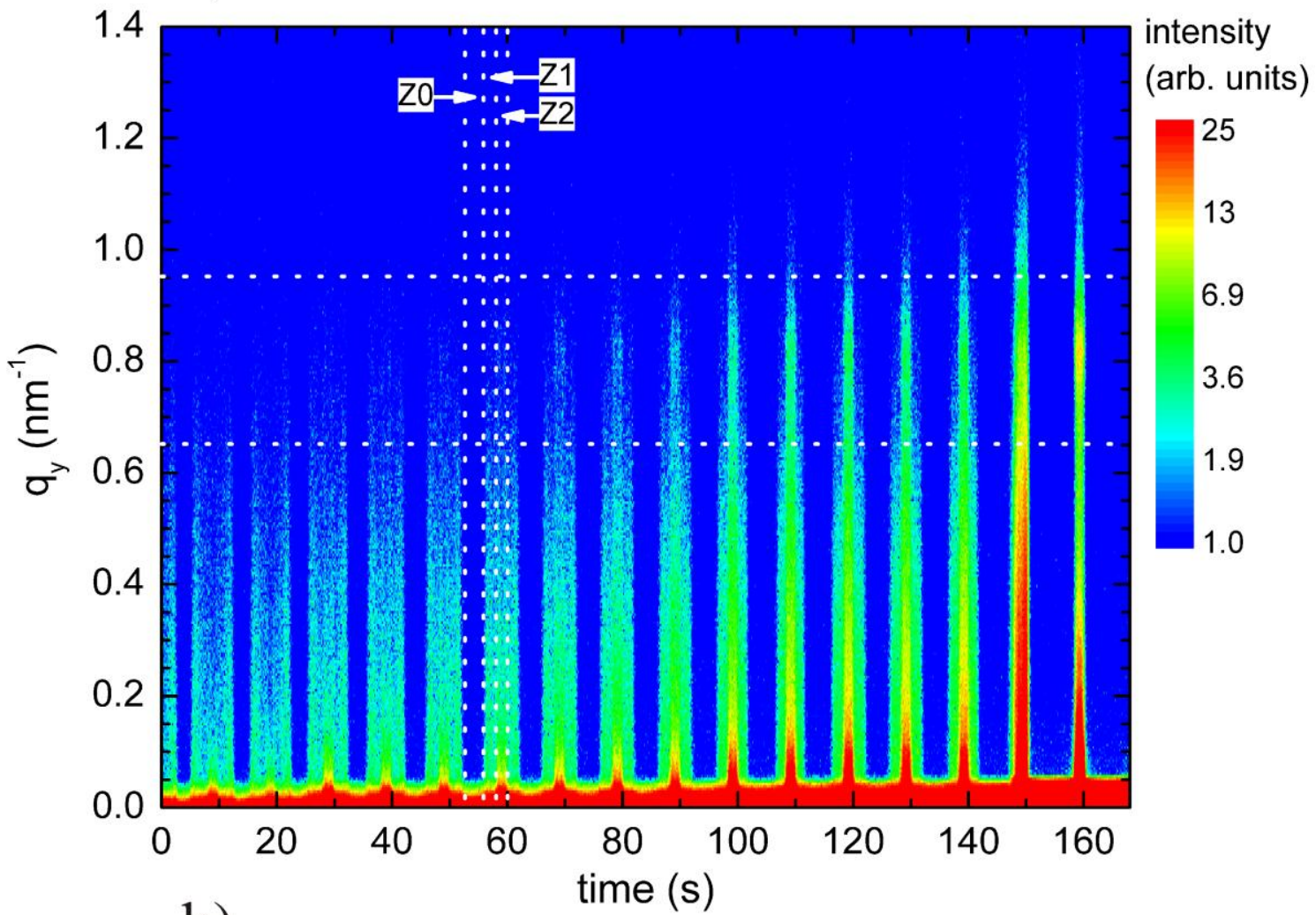

b)

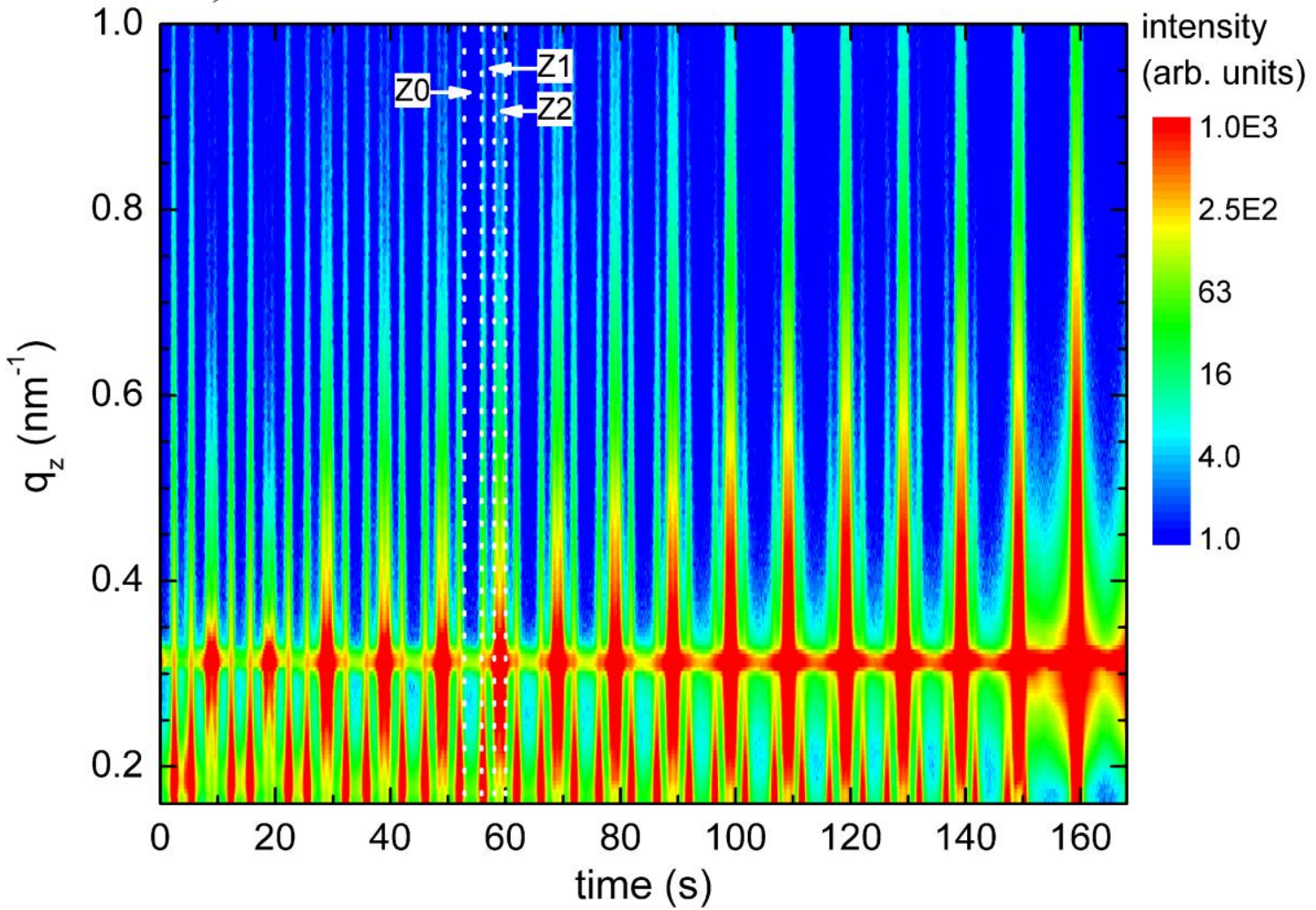

Figure 4. The $t-q_{y}$ (a) and $t-q_{z}$ (b) maps of the drying colloidal drop forming a monolayer in the vertical scanning mode 


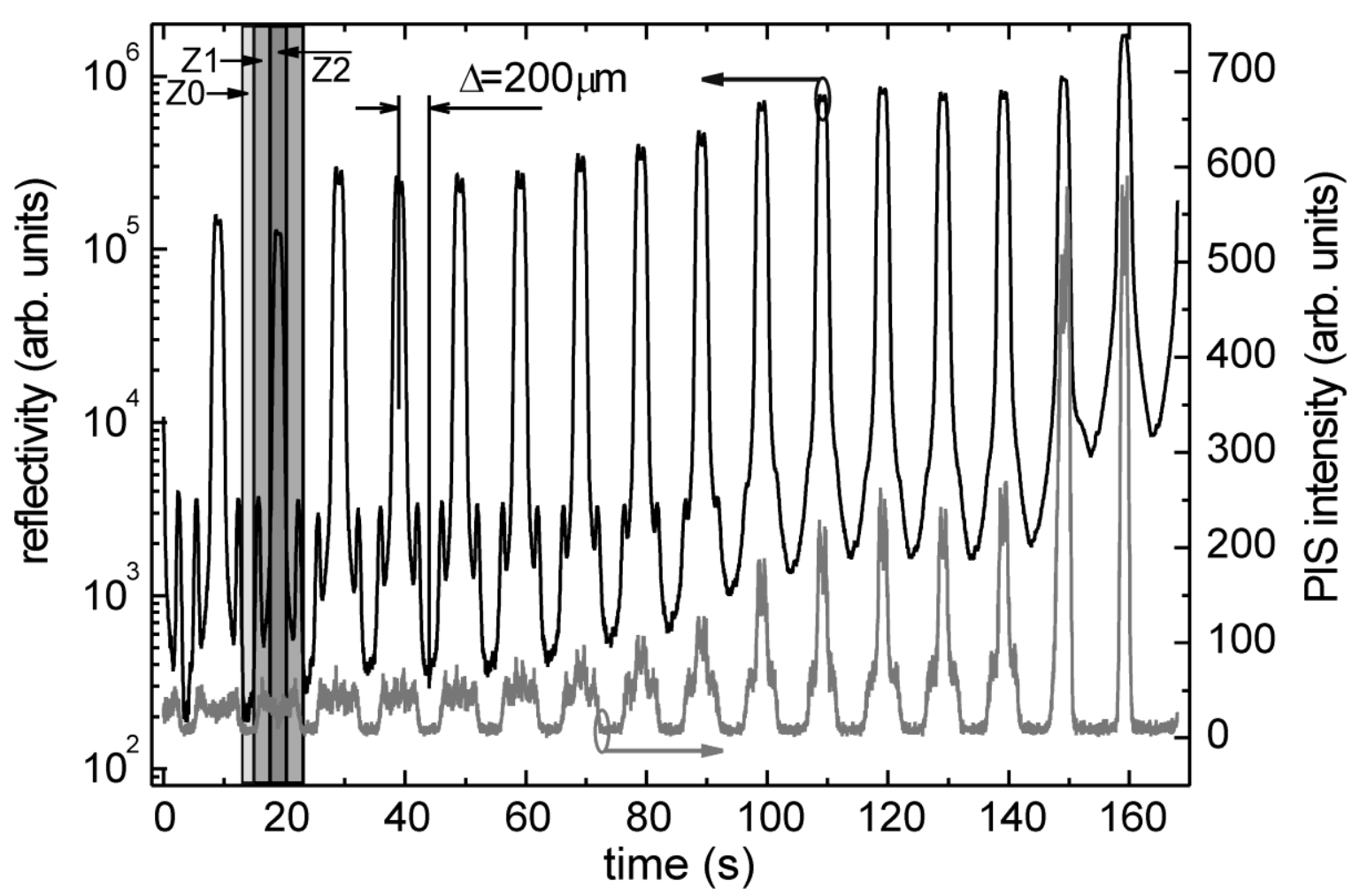

Figure 5. Time-resolved reflectivity (black) and PIS (gray) of the drying colloidal drop in the vertical scanning mode 


\section{sammests small}
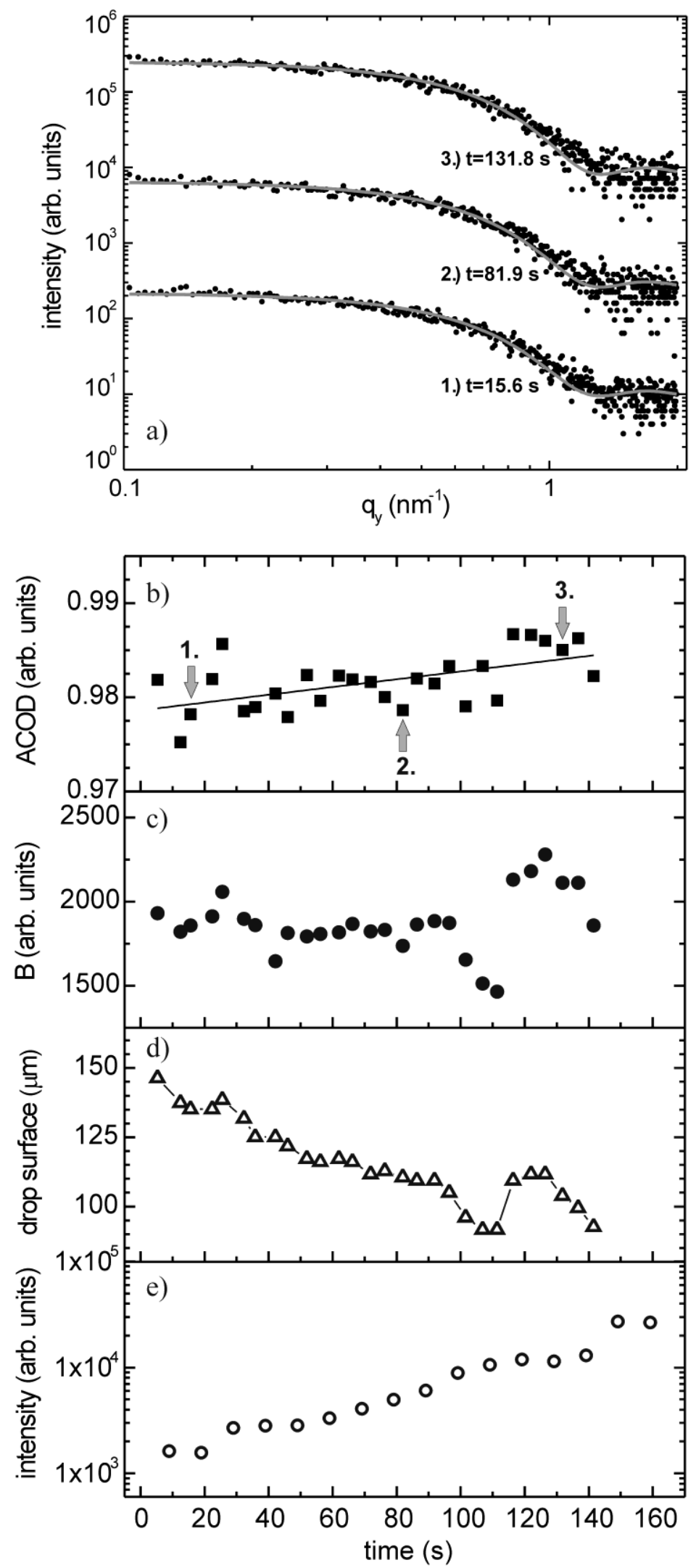

Figure 6. Plots showing a detailed analysis of the scattering events in the zones $\mathrm{Z} 1$ and $\mathrm{Z} 2$ in the vertical scanning mode. Selected scattering profiles integrated inside the zone Z1 (a), the adjusted coefficients of determination (ACOD) (b), $B$ parameter (c), the height of the drop surface above the substrate (d), temporal evolution of PIS in the zone Z2 (e) 


\section{cenomo sameness small}

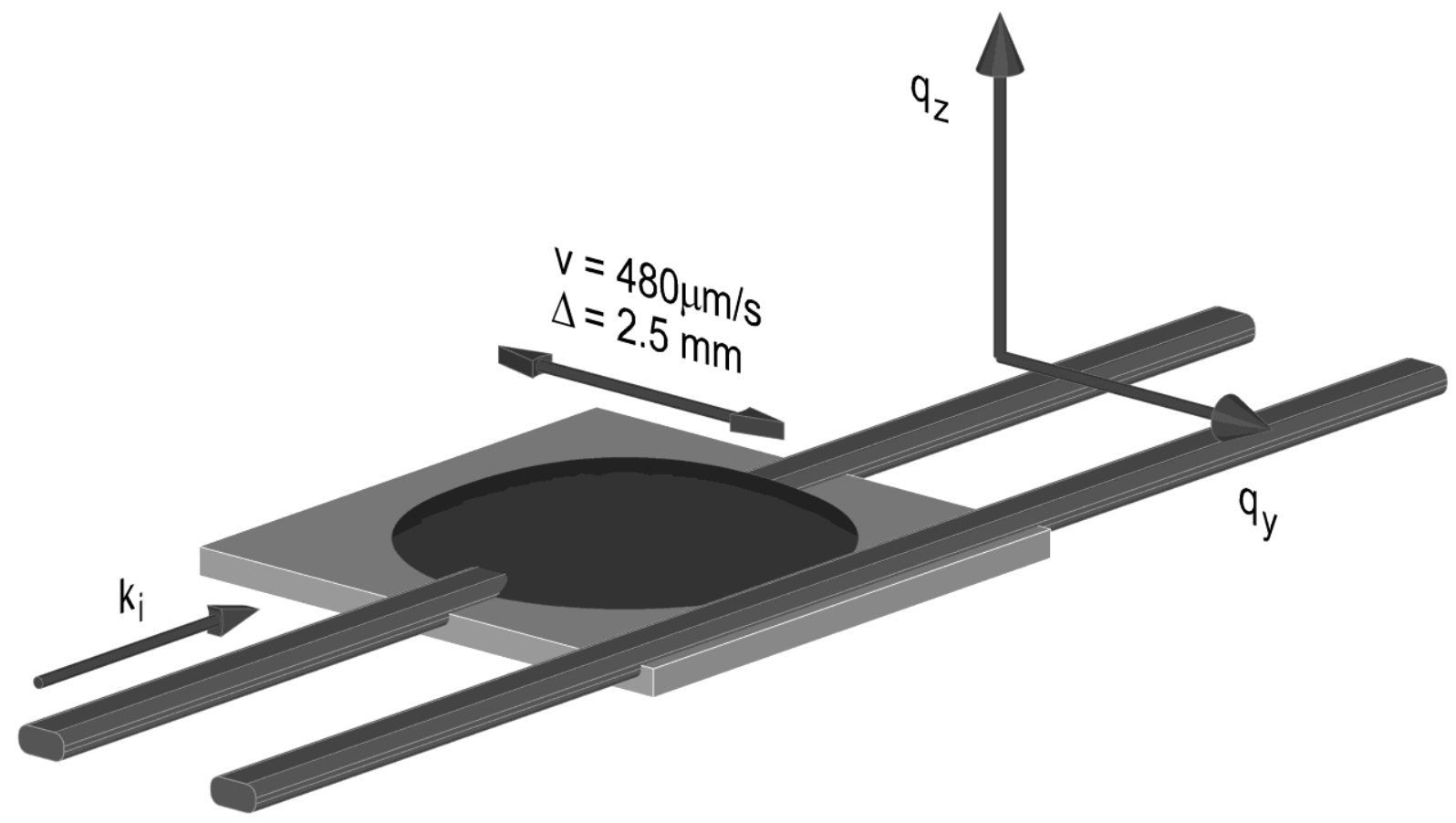

Figure 7. Horizontal scanning scheme 


\section{small}
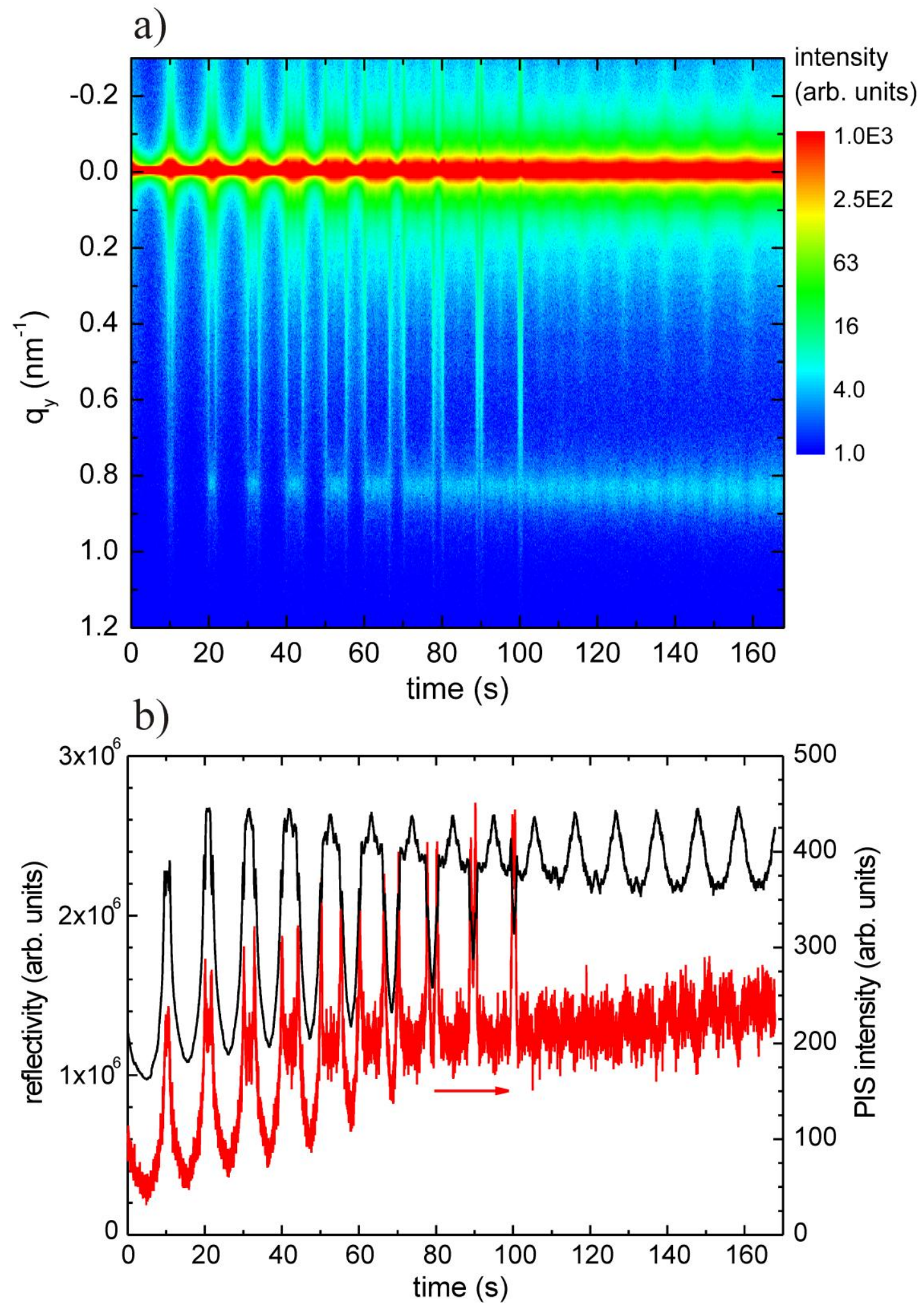

Figure 8. The $t-q_{y}$ map (a) and time-resolved reflectivity (black) and PIS (red) (b) of the drying colloidal drop forming a monolayer in the horizontal scanning mode 
Real-time tracking of nanoparticle self-assembling: The grazing-incidence small angle X-ray scattering technique was employed for an in-situ tracking of the reciprocal space. A focused synchrotron beam, $25 \mathrm{~ms}$ exposure time of X-ray camera and sample oscillations opened the possibility to relate the dynamic reciprocal and direct space features and to localize the nanoparticle self-assembling.

Self-assembly

P. Siffalovic, E. Majkova, L. Chitu, M. Jergel, S. Luby, I. Capek, A. Satka, A. Timmann, and S. V. Roth

Real-time Tracking of Superparamagnetic Nanoparticle Self assembling

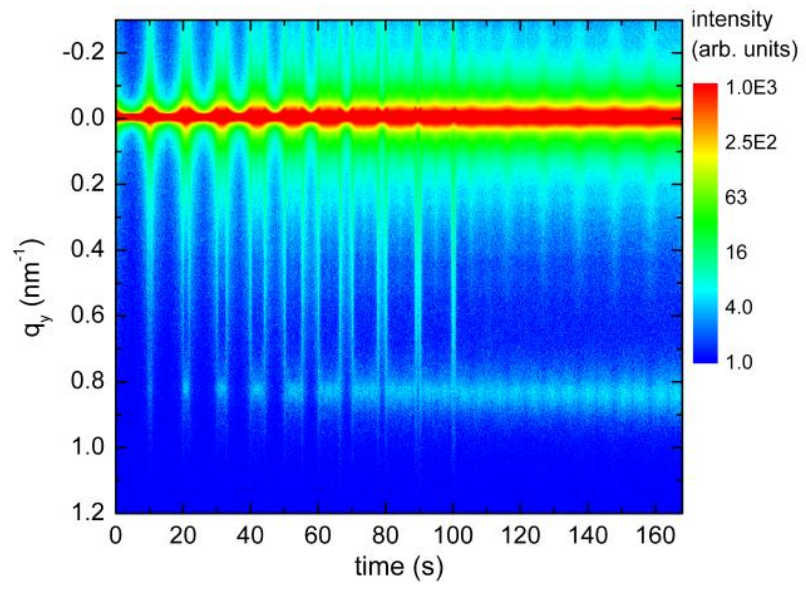

Page Headings

Left page: P. Siffalovic et al.

Right page: Real-time Tracking of Superparamagnetic Nanoparticle Self assembling 